\title{
Analyse biomécanique masticatrice chez des traversodontidés eucynodontes du Trias de Madagascar
}

\author{
Lovasoa RANIVOHARIMANANA \\ Département de Paléontologie et d'Anthropologie biologique, \\ Faculté des Sciences, Université d'Antananarivo (Madagascar) \\ ranivolova@moov.mg
}

MOTS CLÉS

Mastication, muscles adducteurs, mandibule, site occlusal, double leviers.
Ranivoharimanana L. 2012. - Analyse biomécanique masticatrice chez des traversodontidés eucynodontes du Trias de Madagascar. Geodiversitas 34 (3): 505-515. http://dx.doi. org/10.5252/g2012n3a3

\section{RÉSUMÉ}

L'analyse de la biomécanique de la mandibule ne peut s'effectuer sans la compréhension du degré de développement et du type d'arrangement des muscles adducteurs. Chez les cynodontes non mammaliens, le muscle temporal et le masséter superficiel jouent un rôle primordial dans les mouvements de la mâchoire inférieure lors de la transformation alimentaire. Ils constituent ainsi les éléments clés de cette analyse. Des études antérieures sur des cynodontes non mammaliens dont Dadadon isaloi Flynn, Parrish, Rakotosamimanana, Simpson \& Wyss, 1999 et Menadon besairiei Flynn, Parrish, Rakotosamimanana, Simpson \& Wyss, 1999, ont démontré la réalisation, par ces animaux, d'une occlusion dynamique complexe des dents postcanines, inférieures et supérieures. La considération du point de morsure comme second point d'appui au même titre que l'articulation crânio-mandibulaire est le fondement même du système à double leviers. Cette méthodologie a permis de: 1) quantifier la résistance opposée par la nourriture au niveau du site occlusal; et 2) mettre en évidence l'existence d'une charge verticale nette positive, de nature compressive agissant au niveau de l'articulation crânio-mandibulaire chez Dadadon isaloi et Menadon besairiei durant l'interactivité des muscles élévateurs au cours de la mastication. 


\author{
KEY WORDS \\ Mastication, \\ adductor muscles, \\ mandible, \\ occlusal site, \\ bifulcral model.
}

\begin{abstract}
Biomechanical analysis of the jaw apparatus in eucynodont traversodontids from the Triassic of Madagascar.

Mandible biomechanics analysis cannot take place without the understanding of the development degree and the arrangement pattern of the adductor muscles. In the non mammalian cynodonts, the temporal and the superficial masseter muscles play a primordial role in the lower jaw motion during the food processing. They constitute the key elements of this analysis. Previous studies on non mammalian cynodonts including Dadadon isaloi Flynn, Parrish, Rakotosamimanana, Simpson \& Wyss, 1999 and Menadon besairiei Flynn, Parrish, Rakotosamimanana, Simpson \& Wyss, 1999 demonstrated the realization, by these animals, of a complex dynamic occlusion of the lower and superior postcanine teeth. The consideration of the bite point as second occlusal fulcrum equal in status to the cranio-mandibular joint is the basis of the bifulcral model. This methodology allows: 1) to quantify the resistance opposed by food at the level of the occlusal site; and 2) to highlight a positive net vertical load, of compressive nature, acting to the level of the cranio-mandibular joint during the interactivity of the elevator muscles, i.e. during mastication.
\end{abstract}

\section{INTRODUCTION}

Dadadon isaloi Flynn, Parrish, Rakotosamimanana, Simpson \& Wyss, 1999 (Flynn et al. 2000) et Menadon besairiei Flynn, Parrish, Rakotosamimanana, Simpson \& Wyss, 1999 (Flynn et al. 2000; Kammerer et al. 2008) traversodontidés du Trias moyen-supérieur de Madagascar, proviennent de la base de la Formation Isalo II, Bassin de Morondava, sud-ouest de l'île (Besairie 1971). Des études sur des cynodontes (incluant ces taxons) ont démontré la réalisation par ces animaux, d'une occlusion dynamique complexe des dents postcanines inférieures et supérieures à direction postéro-dorsale (Crompton 1972b ; Ranivoharimanana 2007). Chez différents groupes de thérapsides, la modification du type, le degré de développement et l'arrangement de la musculature adductrice semblent concorder avec une réduction des forces générées au niveau de l'articulation crânio-mandibulaire durant les phases de la mastication (Crompton \& Hylander 1986). Selon Bramble (1978), leur articulation crâniomandibulaire subit des charges nettes, soit neutres, soit quelque peu négatives. Kemp (1972) a été le seul à supposer l'existence d'une charge positive opérant au niveau de l'articulation mandibulaire suite à la contraction de la musculature adductrice. Aucune étude chez les synapsides, et particulièrement chez les cynodontes non mammaliens, n'a encore confirmé l'existence d'une charge (tensile ou compressive) agissant au niveau de l'articulation crânio-mandibulaire au cours du cycle masticatoire (Barghusen 1968; Turnbull 1970; Demar \& Barghusen 1972; Rensberger 1973; Bramble 1978; Hiiemae \& Crompton 1985; Crompton 1995; Koolstra \& Van Eijden 1995; Reilly et al. 2001; Goswami et al. 2005; Preuschoft \& Witzel 2005). Déterminer la nature de la résultante des forces agissant au niveau de l'articulation crânio-mandibulaire au cours de la première étape de la transformation alimentaire, constitue donc l'objectif de ce travail. La corrélation fonction, mécanique/structures de la mandibule par le biais de la méthode à double leviers (Bramble 1978) est utilisée ici. Une reconstitution de la musculature adductrice responsable de la fermeture de la mandibule, est proposée dans 

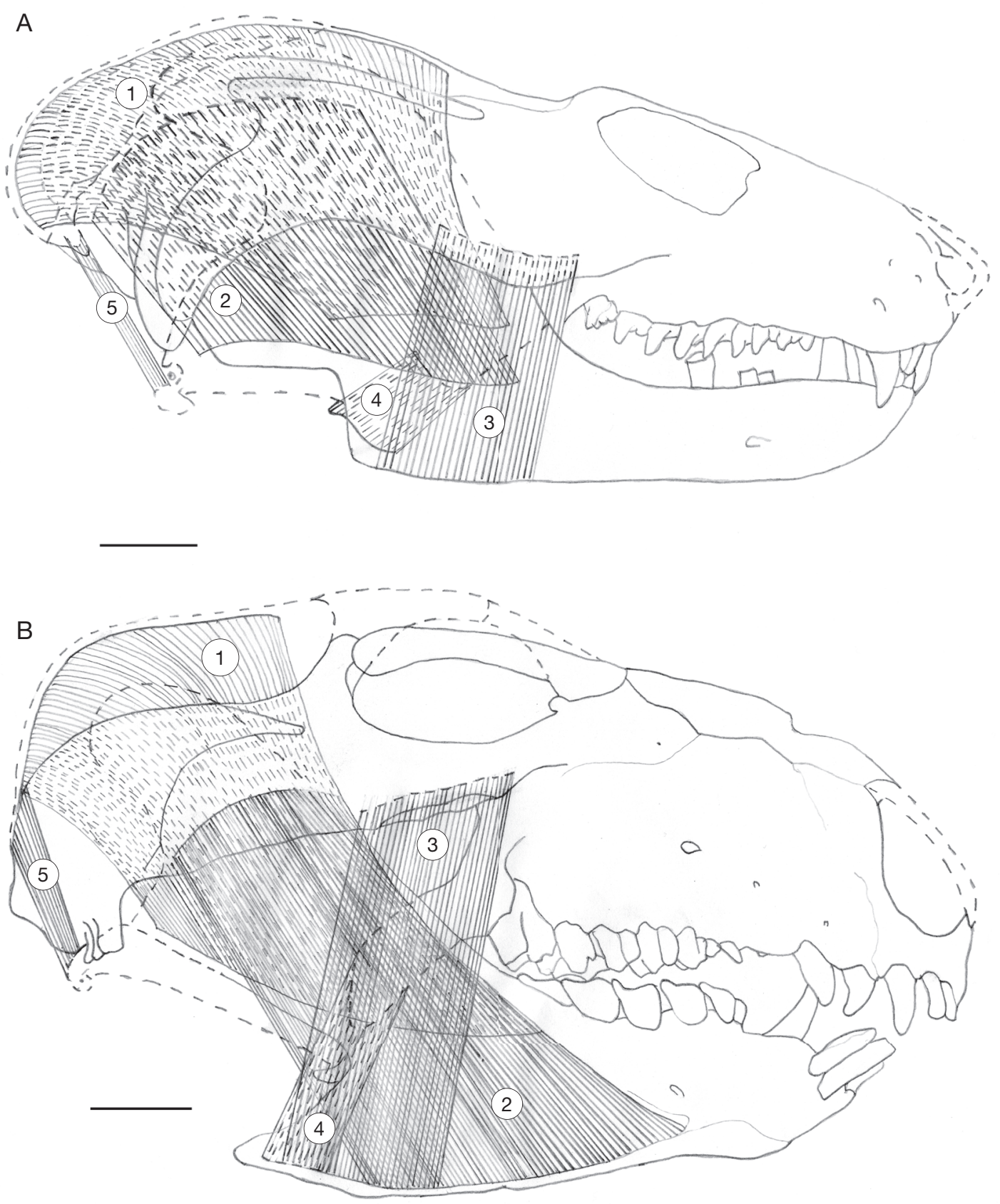

FIG. 1. - Reconstitution de la musculature adductrice chez: A, Dadadon isaloi Flynn, Parrish, Rakotosamimanana, Simpson \& Wyss, 1999 (Ranivoharimanana 2007); B, Menadon besairiei Flynn, Parrish, Rakotosamimanana, Simpson \& Wyss, 1999 (Ranivoharimanana 2007). 1, muscle temporal; 2 , muscle masséter profond; 3 , muscle masséter superficiel; $\mathbf{4}$, muscle ptérygoïdien interne; 5 , muscle dépresseur de la mandibule. Échelles: $2 \mathrm{~cm}$. 

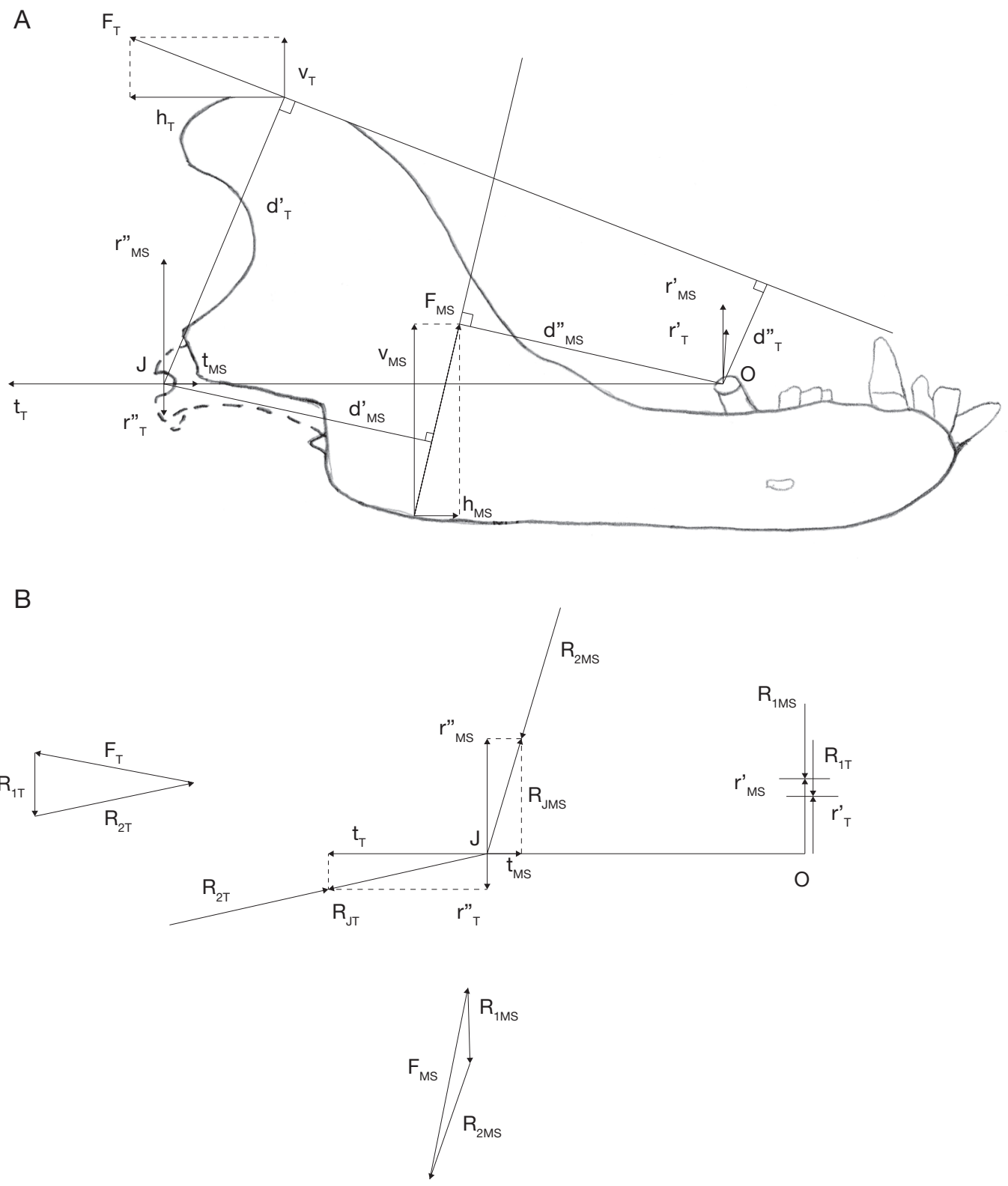

FIG. 2. - Les différentes forces motrices et résistantes au cours de la mastication chez Dadadon isaloi Flynn, Parrish, Rakotosamimanana, Simpson \& Wyss, 1999 (Ranivoharimanana 2007) d'après le modèle à double leviers (Bramble 1978). Abréviations: voir Matériel et méthodes.

ce but. L'état de conservation (absence de la crête occipitale, des os postdentaires, bord postéro-dorsal de l'apophyse coronoïde incomplet) limite cependant l'approche quantitative.

\section{MATÉRIEL ET MÉTHODES}

MATÉRIEL

Le matériel utilisé dans cette étude consiste en: 


\section{A}
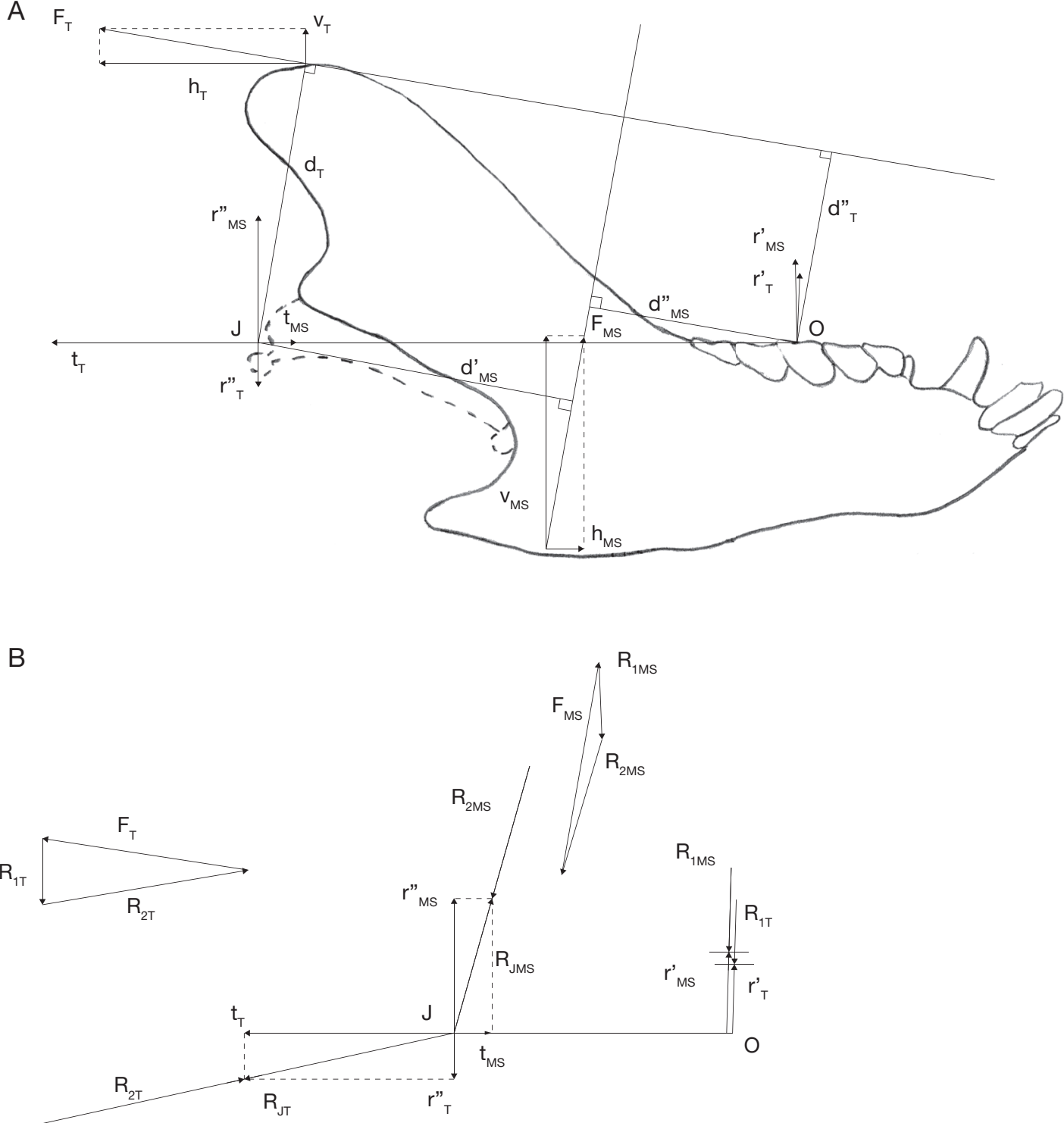

FIG. 3. - Les différentes forces motrices et résistantes au cours de la mastication chez Menadon besairiei Flynn, Parrish, Rakotosamimanana, Simpson \& Wyss, 1999 (Ranivoharimanana 2007) d'après le modèle à double leviers (Bramble 1978). Abréviations: voir Matériel et méthodes.

- l'holotype de Menadon besairiei (UA 10601), MÉTHOde

crâne et mandibule bien conservés (Flynn et al.1999, Morphologie fonctionnelle

2000; Kammerer et al. 2008);

Le fonctionnement de l'appareil masticatoire im- un crâne bien conservé (FMNH-PR 2232) et une hémimandibule droite (UA 10608) de Dadadon isaloi (Flynn et al. 1999, 2000). plique une corrélation entre quatre facteurs, lesquels constituent des indicateurs standards pour l'analyse du mécanisme mandibulaire (Crompton 1963, 
1972a, b, 1995; Barghusen 1968, 1972; Turnbull 1970; Demar \& Barghusen 1972; Bramble 1978; Hiiemae 1978; Kemp 1982; Crompton \& Hylander 1986).

Il s'agit de:

- la morphologie crânienne (particulièrement la crête pariétale, la fenêtre temporale, l'arcade zygomatique, le processus latéral du ptérygoïde);

- les caractéristiques de la mâchoire inférieure (dont l'apophyse coronoïde, la fosse massétérienne, la région angulaire, les os accessoires: surangulaire, angulaire, articulaire, préarticulaire, retroarticulaire).

Ces deux facteurs permettent l'analyse des arrangements et du degré de développement des muscles adducteurs:

- le point de contact entre la postcanine inférieure et supérieure constitue le deuxième point d'appui du système de levier au même titre que le centre d'articulation crânio-mandibulaire;

- l'articulation crânio-mandibulaire, laquelle subit les stress générés durant la mastication.

L'analyse des différentes forces intervenant au niveau de la mandibule est la base de cette étude. Une reconstitution de la musculature adductrice de Dadadon isaloi et de Menadon besairiei est proposée (Fig. 1). L'interactivité des muscles élévateurs génère les forces motrices. En revanche, le crâne (via le carré, le squamosal et le maxillaire) développe les forces de réaction respectivement au niveau de l'articulation crânio-mandibulaire et du point de morsure (Bramble 1978; Koolstra \& Van Eijden 1995).

Chez les traversodontidés, le complexe musculaire jouant un rôle élévateur de la mandibule est composé (Avis 1964; Crompton 1963, 1972a; Barghusen 1968; Turnbull 1970) :

- des muscles adducteurs externes formés par le muscle temporal et le complexe masséter (masséter profond et superficiel);

- du muscle adducteur interne représenté par le muscle ptérygoïdien interne.

Ces muscles représentent le composant dynamique de la géométrie alimentaire, tandis que les dents et les os constituent son composant statique. Ces deux composants forment un système intégré de la géométrie opérationnelle de la mastication (Bramble 1978).

\section{Méthode à double leviers}

Au cours du cycle masticatoire, la mandibule constitue la barre rigide du levier. Celle-ci peut être mobile autour de l'axe fixe «OJ» reliant le point de morsure « $\mathrm{O}$ » et le centre de l'articulation crânio mandibulaire «J». Ces deux points constituent les deux pivots du système. Les muscles adducteurs fonctionnent par paires antagonistes, le muscle temporal couplé avec le masséter profond d'un côté et le masséter superficiel couplé avec le ptérygoïdien interne de l'autre. Le muscle dépresseur de la mandibule, par ses caractéristiques, se joint au premier couple. Il semble jouer un rôle non négligeable dans la mastication. Cependant, de par leur importance majeure au sein de chaque couple, seuls sont considérés ici le muscle temporal et le masséter superficiel. Leur interactivité produit une rotation de levier. Celle-ci dépend de la tension exercée mais surtout du degré de développement du bras de levier, c'est-à-dire essentiellement du moment généré.

Chaque unité musculaire exerce une tension entre son point d'origine et son point d'attachement. Cette tension, représentée par une ligne d'action définie par les centroïdes d'origine et d'insertion, est dirigée vers le point d'origine (Koolstra \& Van Eijden 1995). Sa valeur est assignée proportionnellement à la taille relative du muscle correspondant (Turnbull 1970). Elle se transforme en action mécanique en produisant un mouvement (Elftman 1941). La tension musculaire qui est perpendiculaire à son bras de levier constitue la tension la plus efficace dans l'accomplissement du mouvement de fermeture de la mandibule (Crompton 1963). Les variations dans la direction des fibres composant un muscle n'ont pas d'impact dans le déroulement général du mécanisme mandibulaire (Demar \& Barghusen 1972). En résumé, les mouvements masticatoires sont assurés et contrôlés par la combinaison des activités des muscles masticateurs. La résultante des directions et intensités des lignes d'action des muscles adducteurs interactifs va déterminer le mouvement de la mandibule dans les conditions d'équilibre statique pendant le cycle masticatoire (Bramble 1978).

Pour comparer le mécanisme de l'appareil masticateur des deux spécimens, les crânes et mandibules sont standardisés et schématisés suivant une même longueur (Crompton \& Hylander 1986). 
L'analyse du mécanisme mandibulaire par la méthode à double leviers permet (voir Bramble 1978) : - d'étudier la répartition des forces motrices générées à travers l'appareil masticateur par l'activité du complexe adducteur;

- de démontrer que, sur le plan vertical, les forces de rotation s'opposent au niveau de l'articulation crânio-mandibulaire, annulant ou réduisant la charge qui s'exerce à ce niveau;

- de démontrer que l'équilibre statique doit être acquis pour permettre la mastication.

\section{ABRÉVIATIONS}

Institutions

AMNH American Museum of Natural History (NewYork) ;

UCSB University of California Santa Barbara;

FMNH Field Museum of Natural History (Chicago);

DPAB Département de Paléontologie et d'Anthropologie Biologique (Université d'Antananarivo, Madagascar);

PR Collection de reptiles au FMNH (Chicago);

UA Collection paléontologique de l'Université d'Antananarivo (Madagascar).

\section{Légendes des caractères crânio-mandibulaires}

ACM articulation crânio-mandibulaire;

J centre de l'ACM;

$\mathrm{O}$ site occlusal ;

OJ bras de résistance;

$\mathrm{F}_{\mathrm{T}} \quad$ tension du muscle temporal;

$\mathrm{v}_{\mathrm{T}} \quad$ composant vertical du muscle temporal;

$\mathrm{h}_{\mathrm{T}} \quad$ composant horizontal du muscle temporal;

$\mathrm{d}_{\mathrm{T}} \quad$ bras de levier primaire du muscle temporal;

$\mathrm{d}_{\mathrm{T}} \quad$ bras de levier secondaire du muscle temporal;

$\mathrm{t}_{\mathrm{T}} \quad$ force de translation du muscle temporal;

$\mathrm{r}_{\mathrm{T}}$ force de rotation primaire du muscle temporal;

$\mathrm{r}{ }_{\mathrm{T}} \quad$ force de rotation secondaire du muscle temporal;

$\mathrm{F}_{\mathrm{MS}} \quad$ tension du muscle masséter superficiel;

$\mathrm{v}_{\mathrm{MS}} \quad$ composant vertical du muscle masséter superficiel;

$\mathrm{h}_{\mathrm{MS}} \quad$ composant horizontal du muscle masséter superficiel;

d'MS bras de levier primaire du muscle masséter superficiel;

d" MS bras de levier secondaire du muscle masséter superficiel ;

$\mathrm{t}_{\mathrm{MS}} \quad$ force de translation du muscle masséter superficiel;

r'MS force de rotation primaire du muscle masséter superficiel ; r" MS force de rotation secondaire du muscle masséter superficiel;

$\mathrm{R}_{\mathrm{JT}} \quad$ résultante de $\mathrm{tT}$ et $\mathrm{r}$ ” $\mathrm{T}$;

$\mathrm{R}_{1 \mathrm{~T}} \quad$ résistance développée par le maxillaire vis-àvis de r'T;

$\mathrm{R}_{2 \mathrm{~T}} \quad$ résistance développée par le crâne vis-à-vis de RJT;

$\mathrm{R}_{\mathrm{JMS}} \quad$ résultante de tMS et r"MS ;

$\mathrm{R}_{1 \mathrm{MS}} \quad$ résistance développée par le maxillaire vis-àvis de r'MS;

$\mathrm{R}_{2 \mathrm{MS}} \quad$ résistance développée par le crâne vis-à-vis de RJMS ;

$\mathrm{R}$ force tensile agissant vers le bas au niveau de l'articulaire;

$\mathrm{T} \quad$ tension du muscle temporal;

MS tension du muscle masséter superficiel ;

B force de morsure.

\section{RÉSULTATS}

\section{MORPHOLOGIE FONCTIONNELLE}

Le muscle temporal, plus volumineux chez Menadon besairiei que chez Dadadon isaloi (chez lequel il est étalé en éventail) est composé de fibres orientées très postéro-dorsalement (Fig. 1). Cette grande masse musculaire confère à Menadon besairiei une grande force de contraction et donc une forte capacité préhensile. Compte tenu de son site d'origine et de son aire d'insertion, le masséter profond est orienté postéro-dorsalement de la même manière que le temporal. Corrélativement au développement de la fosse masséterienne, il est moins développé chez Dadadon isaloi que chez Menadon besairiei. Le masséter superficiel, composé de longues fibres verticales chez Menadon besairiei lui confère une grande ouverture de la bouche, favorisant une forte préhension. En revanche, chez Dadadon isaloi, chez qui le crâne est bas, le masséter superficiel est constitué de courtes fibres corrélativement à une faible ouverture de la bouche. Le ptérygoïdien interne, modérément développé, présente une direction verticale presque parallèle à celle du masséter superficiel chez Menadon besairiei. Chez Dadadon isaloi en revanche, il est peu volumineux, formé de courtes fibres, et disposé antéro-dorsalement. Ce dernier trait lui confere une amplitude mécanique moindre par rapport à Menadon besairiei. Faiblement développé par rapport aux autres muscles, le dépresseur de la mandibule est orienté postéro-dorsalement, 
tout comme le temporal et le masséter profond chez les deux espèces. Considérés ensembles par rapport à leur orientation, ces muscles forment deux groupes antagonistes: le temporal avec le masséter profond et le dépresseur de la mandibule à orientation postéro-dorsale d'un côté, puis le masséter superficiel avec le ptérygoïdien interne à orientation dorsale de l'autre. Ainsi, ce dernier couple constitue le groupe dominant. C'est une des caractéristiques des herbivores (Crompton 1963; Bramble 1978).

\section{ANALYSE BIOMÉCANIQUE DE LA MANDIBULE}

PAR LA MÉTHODE À DOUBLE LEVIERS

L'analyse des différentes forces motrices mises en jeu au cours d'un cycle masticatoire respectivement chez Menadon besairiei et Dadadon isaloi démontre que le muscle temporal opère en mode négatif et libère une force verticale négative orientée vers le bas au niveau du point J. Cette charge verticale a tendance à écarter la mandibule du crâne. De son côté, le muscle masséter superficiel opère en mode positif et produit en J une force de rotation secondaire positive, orientée vers le haut et largement supérieure à celle générée par le temporal. Elle a tendance à presser la mandibule contre le crâne. Leur résultante serait alors une charge positive de nature compressive. Comparativement, les charges verticales nettes positives intervenant au niveau de l'articulation crânio-mandibulaire des deux espèces sont différentes: celle de Menadon besairiei est légèrement plus grande que celle de Dadadon isaloi. Ceci suggère que, pour Menadon besairiei, l'articulation crânio-mandibulaire est plus robuste que celle de Dadadon isaloi. Par conséquent, Menadon besairiei aurait une mastication plus énergique, suggérant une alimentation à base de matière végétale plus coriace. La force négative générée par la contraction du muscle temporal, associée à celles produites par le masséter profond et le dépresseur de la mandibule, est contrebalancée par la résultante des forces positives délivrées par les activités du masséter superficiel et du ptérygoïdien interne. Les deux rôles joués par le dépresseur de la mandibule, abaisseur et stabilisateur des charges opérationnelles au niveau de l'articulation crânio-mandibulaire (Bramble 1978), sont donc ici confirmés.
Lanalyse des tensions au niveau des points $\mathrm{O}$ et $\mathrm{J}$ au cours d'une morsure est représentée respectivement Figures 2 (chez Dadadon isaloi) et 3 (chez Menadon besairiei). Chaque figure présente deux sections, identifiées par les lettres A et B. Les forces motrices (décomposées en forces de translation et de rotation, primaires et secondaires) ainsi que les forces de réaction correspondantes sont rassemblées section $\mathrm{B}$ en conditions d'équilibre statique. Ainsi, géométriquement parlant, toutes les forces motrices et résistantes forment un polygone fermé.

\section{DISCUSSION}

Opérant positivement par rapport au crâne, à l'encontre du carré, la charge verticale est à effet désarticulant. La réalisation d'une occlusion bilatérale à direction postéro-dorsale (Goswami et al. 2005; Ranivoharimanana 2007), implique une articulation stable et robuste entre le carré et l'articulaire. Cependant chez les cynodontes, le carré est streptostylique et petit par rapport aux os post-dentaires (articulaire compris). Ces caractères entraînent l'affaiblissement de l'articulation crâniomandibulaire qui ne peut résister à la force occlusive des dents postcanines durant la transformation des aliments. Ainsi, la charge verticale à ce niveau doit être réduite, sinon nulle. Ceci peut s'effectuer de deux manières :

- par l'apparition d'une articulation additionnelle entre squamosal et surangulaire qui répartit la charge du carré (Romer 1970; Crompton 1972a; Allin \& Hopson 1992; Ranivoharimanana 2007);

- par l'arrangement des muscles adducteurs réduisant au minimum la charge de l'articulation mandibulaire (Crompton 1963, 1989; Bramble 1978; Crompton \& Parker 1978; Crompton \& Hylander 1986).

Le deuxième cas nécessite un équilibre entre forces de rotation secondaire positive et négative sur le plan vertical, équilibre réalisé par la contraction synchrone du muscle temporal et du masséter superficiel. Cette contraction est restreinte à un seul site occlusal qui est représenté par le point d'équilibre (Bramble 1978) où les forces de rotation sont égales, c'est-à-dire où l'articulation 
crânio-mandibulaire n'est sujette à aucune force de rotation, cas des mammiferes. Ce site est matérialisé par l'intersection des lignes d'action projetées antagonistes. Chez Dadadon isaloi et Menadon besairiei (comme chez d'autres cynodontes, cf. Crompton 1963), cette intersection est postérieure à la série dentaire (Figs 2-4) et l'équilibre du site occlusal n'est pas atteint, la charge verticale de l'articulation crânio-mandibulaire ne pouvant être nulle. Mais cette intersection étant proche des postcanines postérieures, le site occlusal le plus postérieur correspond à l'endroit où la contraction synchrone des deux principaux adducteurs s'effectue. Apparemment, chez les autres cynodontes non mammaliens, il n'y a aucun site occlusal d'équilibre et l'articulation crânio-mandibulaire est donc sujette à une charge verticale nette positive d'intensité variable suivant la nourriture consommée.

Chez Diarthrognathus Crompton, 1963 cependant (Fig. 4E), le site occlusal le plus postérieur coïncide avec l'intersection des lignes d'action projetées des deux muscles adducteurs. À la différence des autres cynodontes non mammaliens, son articulation crânio-mandibulaire ne subit pas une charge nette positive.

\section{CONCLUSION}

Similaires aux autres traversodontidés, Dadadon isaloi et Menadon besairiei réalisent, au cours de la mastication, une occlusion dynamique complexe à direction postérodorsale (Ranivoharimanana 2007). L'interactivité des principaux muscles adducteurs, temporal et masséter superficiel, est responsable de la fermeture de la mandibule, donc du mécanisme mandibulaire. Au cours de la transformation alimentaire, la nourriture oppose une certaine résistance à la force de morsure. Ainsi, la considération du point occlusal comme second point d'appui, en plus de l'habituel point d'articulation crânio mandibulaire (méthode à double leviers), a mis en évidence l'existence d'une charge verticale nette positive, de nature compressive au niveau du carré. Il s'agit de la résultante des forces rotationnelles libérées par la contraction des muscles adducteurs antagonistes, temporal et masséter superficiel. Cette
A

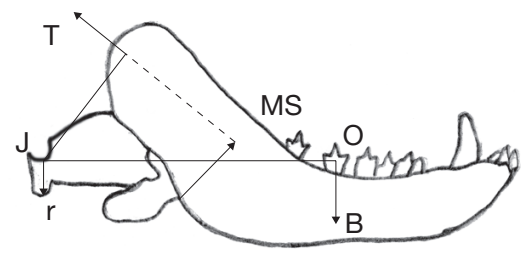

B

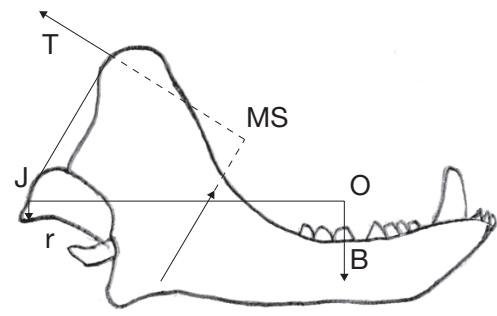

C

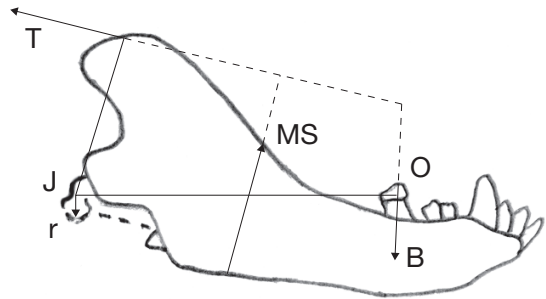

D.

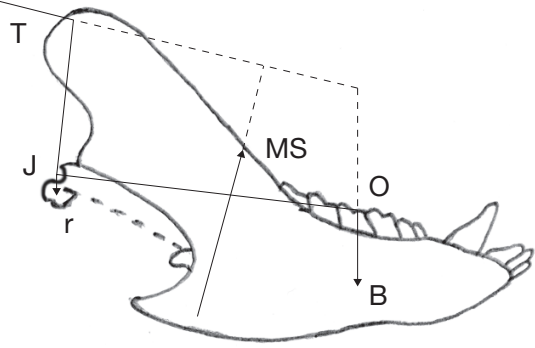

E

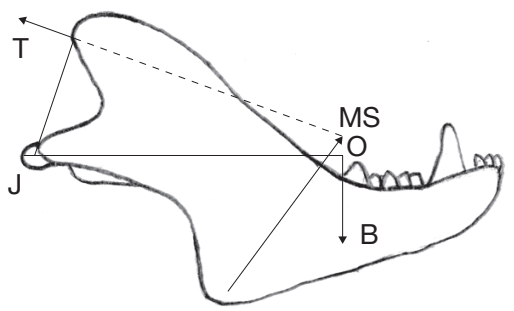

FIG. 4. - Mandibules de cynodontes non mammaliens en vue latérale, montrant la position de l'intersection des lignes d'action projetées du temporal (T) et du masséter superficiel (MS) postérieure à la série dentaire: A, Thrinaxodon; B, Trirachodon; C, Dadadon isaloi; D, Menadon besairiei; E, Diarthrognathus. A, B, E : Crompton 1963. 
contraction a tendance à comprimer la mandibule contre le crâne. Comparativement, celle de Menadon besairiei est légèrement plus grande que celle de Dadadon isaloi. L'articulation crânio mandibulaire de Menadon besairiei serait alors plus robuste, pouvant faire face à une mastication plus énergique, suggérant une consommation de fibres végétales plus coriaces.

\section{Remerciements}

Je suis infiniment reconnaissante aux principaux inspirateurs de ce travail, John Flynn et André Wyss (AMNH et UCSB, USA). Mes sincères remerciements s'adressent aux rapporteurs Bernard Battail et J. Sébastien Steyer (MNHN et CNRS, Paris) dont le dévouement a beaucoup contribué à la finalisation de cet article, ainsi qu'à Annamarie Ohler (MNHN). Je tiens également à remercier la National Geographic Society et le FMNH d'une part, le Ministère de l'Enseignement Supérieur et de la Recherche Scientifique, le Ministère de l'Énergie et des Mines, la Présidence de l'Université d'Antananarivo, et la Faculté des Sciences de Madagascar d'autre part, d'avoir facilité les travaux de terrain et de laboratoire. Je ne saurais oublier d'adresser ma reconnaissance à R. Andriantompohavana, L. Razafimanantsoa, A. Toto Volahy, J. A. Rabarison, A. Gandie et à P. Vavisoro (Université d'Antananarivo) ; à J. James Hopson (Université de Chicago), Robin Wathley, Gina Westhley, Elaine Zeiger, William Simpson, Lorie Barber, Steve Goodman (FMNH) pour leur assistance durant les travaux de terrain ou lors de mon stage au FMNH; à Bango Ramangarisoa (Faculté des Sciences) pour la mise en forme des illustrations.

\section{RÉFÉRENCES}

Allin E. F. \& Hopson J. A. 1992. - Evolution of the auditory system in Synapsida ("mammal-like reptiles" and primitive mammals) as seen in the fossil record, in Webster D. B., Fay R. F. \& Popper A. N. (éds), The Evolutionary Biology of Hearing. Springer-Verlag, New-York, Berlin, Heidelberg, London, Paris, Tokyo, Hong Kong, Barcelone, Budapest: 587-614.
Avis V. 1964. - The significance of the angle of the mandible: an experimental and comparative study. American Journal of Physical Anthropology 19 (1): 55-61.

BARGHUSEN H. R. 1968. - The lower jaw of Cynodonts (Reptilia, Therapsida) and the evolutionary origin of mammal-like adductor jaw musculature. Postilla Yale Peabody Museum of Natural History 116: 1-49.

BARGHUSEN H. R. 1972. - The origin of the mammalian jaw apparatus, in SCHUMACHER G. H. (éd.), Morphology of the Maxillomandibular Apparatus. VEB Georg Thieme, Leipzig: 26-43, 3 figs.

Besairie H. 1971. — Géologie de Madagascar. I. Les terrains sédimentaires. Annales géologiques de Madagascar 35: 1-463, 89 pls.

BRAMBLE D. M. 1978. - Origin of the mammalian feeding complex: models and mechanisms. Paleobiology 4: 271-301.

Crompton A. W. 1963. - On the lower jaw of Diarthrognathus and the origin of the mammalian lower jaw. Proceedings of the Zoological Society of London 140: 697-750.

Crompton A. W. 1972a. - The evolution of the jaw articulation of cynodonts. Studies in Vertebrate Evolution 17: 231-251.

Crompton A. W. 1972b. - Postcanine occlusion in cynodonts and Tritylodontids. Bulletin of the British Museum of Natural History (Geology) 21: 27-71.

CROMPTON A. W. 1989. - The evolution of mammalian mastication, in WAKE B. D. \& RoTH G. (éd.), Complex Organismal Functions: Integration and Evolution in Vertebrates. John Wiley \& Sons, Berlin: 23-40.

CROMPTON A. W. 1995. - Masticatory function in no mammalian cynodonts and early mammals, in Thomason J. (éd.), Functional Morphology in Vertebrate Paleontology. Cambridge University Press, Cambridge: 55-75.

Crompton A.W. \& Parker P. 1978. - Evolution of the mammalian masticatory apparatus. American Scientist 66: 192-201.

Crompton A. W. \& Hylander W. L. 1986. — Changes in mandibular function following the acquisition of a dentary-squamosal jaw articulation, in HOTTON III N., Maclean P. D., Roth J. J. \& Roth E. C. (éds), The Ecology and Biology of Mammal-like Reptiles 263-282.

DEMAR R. \& BARGHUSEN H. R. 1972. - Mechanics and the evolution of the synapsid jaw. Evolution 26: 622637 (published by Society for the study of evolution).

ELFTMAN H. 1941. - The action of muscles in the body. Biological Symposia 3: 191-209.

Flynn J. J., Parrish J. M., Rakotosamimanana B., Simpson W. F. \& Wyss A. R. 1999. — New Eucynodonts from the early Mesozoic of Madagascar. Journal of Vertebrate Paleontology 19: 45A.

Flynn J. J., Parrish J. M., Rakotosamimanana B., Ranivoharimanana L., Simpson W. F. \& Wyss A. R. 2000. — New traversodontids (Synapsida: Eucy- 
nodontia) from the Triassic of Madagascar. Journal of Vertebrate Paleontology (rapid communication) 20 (3): 422-427.

Goswami A., Flynn J. J., Ranivoharimanana L. \& Wyss A. 2005. - Dental microwear in Triassic amniotes: implications for paleoecology and masticatory mechanics. Journal of Vertebrate Paleontology 25 (2): 320-329.

HiIEMAE K. M. 1978. - Mammalian mastication: a review of the activity of the jaw muscles and the movements they produce in chewing, in BUTLER P. M. \& Joysey K. A. (éds), Development, Function and Evolution of Teeth. Academic Press, London: 359-398.

Hitemae K. M. \& Crompton A. W. 1985. - Mastication food transport and swallowing, in HiLDEBRAND M., Bramble D. M. , Liem K. F. \& WaKe D. B. (éds), Functional vertebrate morphology. The Belknap Press of Harvard University Press, Cambridge: 262-290.

Kammerer C. F., FLYNn J. J., RANivoharimanana L. \& Wyss A. R. 2008. - New material of Menadon besairiei (Cynodontia: Traversodontidae) from the Triassic of Madagascar. Journal of Vertebrate Paleontology 28 (2): 445-462.

KemP T. S. 1972. - Waitsiid Therocephalia and the origin of cynodonts. Cambridge University Museum of Zoology. Vol. 264, no. 857: 1-54.

KEMP T. S. 1982. - Mammal-like Reptiles and the origin of Mammals. Academic press, University Museum and
Department of Zoology, 362 p.

Koolstra J. H. \& VAn EIjDEn T. M. G. J. 1995. Biomechanical analysis of jaw-closing movements. Journal of dental research 74 (9): 1564-1570.

Preuschoft H. \& Witzel U. 2005. - Functional shape of the skull in vertebrates: which forces determine skull morphology in lower primates and ancestral synapsids? The anatomical record part A 283A: 402-413.

RANIVOHARIMANANA L. 2007. - Structures crânio-dentaires: implication bioméchanique de la mandibule de deux nouveaux traversodontidés eucynodontes ("ReptilesMammaliens") du Trias de Madagascar. Thèse de Doctorat, département de Paléontologie et d'Anthropologie Biologique, Université d'Antananarivo, Antananarivo, 219 p.

Reilly S. M., Mcbrayer L. D. \& White T. D. 2001. Prey processing in Amniotes: biomechanical and behavioural patterns of food reduction. Comparative Biochemistry and Physiology Part A 128: 397-415.

RENSBERGER J. M. 1973. - An occlusion model for mastication and dental wear in herbivorous mammals. Journal of Paleontology 47 (3): 515-528.

Romer A. S. 1970. - The Chanares (Argentina) Triassic reptile fauna. VI. A chiniquodontid cynodonts with an incipient squamosal-debtary jaw articulation. Museum Comparative Zoology 344: 1-18.

Turnbull W. D. 1970. - Mammalian masticatory apparatus. Fieldiana Geology 18: 153-356.

Soumis le 24 novembre 2009; accepté le 6 avril 2011. 